\title{
Homozygous missense variant in the human CNGA3 channel causes cone-rod dystrophy
}

\author{
Rehan S Shaikh ${ }^{1}$, Peggy Reuter ${ }^{2}$, Robert A Sisk ${ }^{3}$, Tasleem Kausar ${ }^{1}$, Mohsin Shahzad $^{4}$, Muhammad I Maqsood ${ }^{1}$, \\ Ateeq Yousif ${ }^{5}$, Muhammad Ali ${ }^{1}$, Saima Riazuddin ${ }^{4}$, Bernd Wissinger ${ }^{2}$ and Zubair M Ahmed ${ }^{\star, 1,4}$
}

We assessed a large consanguineous Pakistani family (PKAB157) segregating early onset low vision problems. Funduscopic and electroretinographic evaluation of affected individuals revealed juvenile cone-rod dystrophy (CRD) with maculopathy. Other clinical symptoms included loss of color discrimination, photophobia and nystagmus. Whole-exome sequencing, segregation and haplotype analyses demonstrated that a transition variant (c.955T>C; p.(Cys319Arg)) in CNGA3 co-segregated with the CRD phenotype in family PKAB157. The ability of CNGA3 channel to influx calcium in response to agonist, when expressed either alone or together with the wild-type CNGB3 subunit in HEK293 cells, was completely abolished due to p.Cys319Arg variant. Western blotting and immunolocalization studies suggest that a decreased channel density in the HEK293 cell membrane due to impaired folding and/or trafficking of the CNGA3 protein is the main pathogenic effect of the p.Cys319Arg variant. Mutant alleles of the human cone photoreceptor cyclic nucleotide-gated channel (CNGA3) are frequently associated with achromatopsia. In rare cases, variants in CNGA3 are also associated with cone dystrophy, Leber's congenital amaurosis and oligo cone trichromacy. The identification of predicted p.(Cys319Arg) missense variant in CNGA3 expands the repertoire of the known genetic causes of CRD and phenotypic spectrum of CNGA3 alleles.

European Journal of Human Genetics (2015) 23, 473-480; doi:10.1038/ejhg.2014.136; published online 23 July 2014

\section{INTRODUCTION}

Cone-rod dystrophy $(\mathrm{CRD})^{1}$ is a group of rare, genetically and phenotypically heterogeneous retinal disorders that primarily affect cone photoreceptor function, with concomitant or subsequent loss of rod photoreceptors. Other visual deficits observed in individuals suffering from CRD include color vision abnormalities, maculopathy, photophobia and night blindness. The prevalence of CRD has been estimated to be 1:30000-1:40000. ${ }^{1-3}$ CRD can manifest under a variety of inheritance models, though the autosomal recessive mode of inheritance is the most prevalent. To date, eight genes responsible for autosomal recessive CRD have been identified: ABCA4 (OMIM\#601691), ${ }^{4}$ ADAM9 (OMIM\#602713), ${ }^{5}$ C8orf37 (OMIM\#614477), ${ }^{6}$ CERKL (OMIM\#608381), ${ }^{7}$ EYS (OMIM\#612424), ${ }^{8}$ RPGRIP1 (OMIM\#605446), ${ }^{9}$ RAB28 (OMIM\#612994) ${ }^{10}$ and TULP1 (OMIM\#602280). ${ }^{11}$ However, the known variants do not account for all cases of CRD. ${ }^{12,13}$

Here, we report the genetic cause of juvenile CRD with maculopathy segregating in an autosomal recessive pattern in family PKAB157. Through whole-exome sequencing, we identified a novel variant (c. $955 \mathrm{~T}>\mathrm{C}$ ) in the $C N G A 3$ gene co-segregating with $\mathrm{CRD}$ phenotype in family PKAB157. To date, 81 CNGA3 alleles have been documented in various populations around the world. The majority of these (76) are associated with achromatopsia (OMIM\#600053), while 3 variants are known to cause cone dystrophy $(\mathrm{CD}),{ }^{14}$ one has been reported in a subject diagnosed with Leber's congenital amaurosis (LCA), ${ }^{15}$ and one has been reported in an individual with oligocone trichromacy. ${ }^{16}$ The c.955T $>\mathrm{C}$ change identified in family PKAB157 represents the first variant of CNGA3 which was found to be responsible for the CRD phenotype.

\section{MATERIALS AND METHODS}

Family participation and clinical evaluation

The study conformed to the tenets of the Declaration of Helsinki and received approval from the Institutional Review Board (IRB) Committees at the Children's Hospital Research Foundation, Cincinnati and the Bahauddin Zakariya University, Multan, Pakistan. Informed written consent was obtained from the adult subjects and the parents of minor subjects. The clinical diagnoses were based on ophthalmic examinations, including funduscopy, full-field electroretinography (ERG) recordings, visual acuity measurements, standard color vision testing (Farnsworth Dichotomous Series: D-15) and optical coherence tomography (OCT) scans. Peripheral blood samples were collected from each participating individual for genomic DNA extraction using standard procedures.

\section{Targeted capture and exome sequencing}

In all, $1 \mu \mathrm{g}$ of double-stranded DNA from two affected individuals (IV:1 and IV:4) from family PKAB157 was sheared via sonication on a Diagenode Bioruptor (Diagenode Inc., Denville, NJ, USA). Library construction was performed in an automated manner on an IntegenX Apollo324 (IntegenX, Pleasanton, CA, USA). Following PCR amplification, $1 \mu \mathrm{g}$ of the genomic library was recovered for exome enrichment using the NimbleGen EZ Exome V2 kit (Roche Diagnostics, San Francisco, CA, USA). One hundred base pair paired-end sequencing was performed using the Illumina Hi-Seq 2000 system (Illumina, San Diego, CA, USA). The obtained sequencing data were analyzed

${ }^{1}$ Institute of Molecular Biology and Biotechnology, Bahauddin Zakariya University, Multan, Pakistan; ${ }^{2}$ Molecular Genetics Laboratory, Institute for Ophthalmic Research, University Clinics Tuebingen, Tuebingen, Germany; ${ }^{3}$ Division of Pediatric Ophthalmology, Cincinnati Children's Hospital Research Foundation, Cincinnati, OH, USA; ${ }^{4}$ Department of Otorhinolaryngology Head and Neck Surgery, School of Medicine, University of Maryland, Baltimore, MD, USA; ${ }^{5}$ nstitute of Ophthalmology, Mayo Hospital, King Edward Medical University, Lahore, Pakistan

${ }^{*}$ Correspondence: Dr ZM Ahmed, Department of Otorhinolaryngology Head and Neck Surgery, School of Medicine, University of Maryland, BioPark Building 1, 800 West Baltimore Street, Room 404, Baltimore, MD 21201, USA. Tel: +1 301814 4431; Fax: +1 410706 3090; E-mail: zahmed@smail.umaryland.edu

Received 6 September 2013; revised 6 June 2014; accepted 18 June 2014; published online 23 July 2014 
following the guidelines outlined in the Broad Institute's Genome Analysis Toolkit. ${ }^{17,18}$ The raw data were mapped using the Burrows Wheeler Aligner. ${ }^{18}$ Variants were called using the Unified Genotyper software (Broad Institute, Cambridge, MA, USA), and the data were then subjected to further processing and quality control. ${ }^{17,18}$

To narrow down the potential candidate variants, we used the following filtration technique: (1) Given that we were searching for variants present in a homozygous manner in samples from affected individuals, the disease-causing allele should be present in $100 \%$ of reads. Nevertheless, considering the limitations of this technology (poor sequencing toward the ends of reads and general, minor sequencing errors), ${ }^{19,20}$ we set this limit to $70 \%$. (2) Known SNPs (db135 version) and variants recurring in the 1000 Human Genomes or NHLBI exome databases with a minor allele frequency of $>0.01$ underwent bioinformatic filtration. ${ }^{21,22}$ (3) Non-conserved deep intronic changes, other than those present in predicted regulatory/promoter regions or at splice junctions, were filtered out. (4) Synonymous changes, other than those present at splice junctions, were also filtered out. (5) Pakistani-specific polymorphisms were filtered out using our in-house exome sequencing data of Pakistani individuals with normal vision.

\section{Allelic variant segregation and haplotype analyses}

Primers (Forward: $5^{\prime}$-ttaatggtcagtgataccaacagg- $3^{\prime}$; Reverse: $5^{\prime}$-ctacgaccacaaagagatactcctc- $3^{\prime}$ ) were designed using Primer3 (http://primer3.sourceforge.net/) for $\mathrm{c} .955 \mathrm{~T}>\mathrm{C}$ variant segregation analysis in family PKAB157. The sequencing method applied to the PCR products has been described previously. ${ }^{23} \mathrm{An}$ ABI 3730xl DNA capillary analyzer (Grand Island, NY, USA) was used to resolve the products, and the Lasergene DNAstar software (http://www.dnastar.com) was employed to analyze the results. The c.955T $>$ C sequencing data have been submitted to the NCBI repository (GenBank KJ650082) and LOVD database (http://grenada.lumc.nl/LOVD2/eye/home.php?select_db=CNGA3). Control DNA samples from an ethnically matched Pakistani population were sequenced to detect the mutant allele of CNGA3.

For haplotype analysis in family PKAB157, we genotyped CNGA3-linked short tandem repeat (STR) markers, D2S113, D2S2222, D2S2311, D2S2187 and D2S2209, using genomic DNA from all of the participating affected and unaffected members with an ABI 3730xl DNA capillary analyzer. Alleles were assigned using the Genescan and Genotyper software (Applied Biosystems, Foster City, CA, USA)

\section{Logarithm of odds score calculations}

The marker order and map distances came from the Marshfield genetic map (http:research.marshfieldclinic.org/). Two-point logarithm of odds (LOD) base 10 scores were calculated with MLINK. ${ }^{24}$ We assumed a recessive mode of inheritance, with full penetrance of CRD in homozygotes and no phenocopies. The disease allele frequency was set at 0.001 , with equal meiotic recombination frequencies for males and females.

\section{Bioinformatic analysis}

Four bioinformatic programs, Polyphen-2, ${ }^{25}$ SNPs $3 \mathrm{D},{ }^{26}$ MutationTaster $^{27}$ and the Project HOPE web server, ${ }^{28}$ were used to determine the predicted effect of the novel CNGA3 missense variant.

\section{Expression of wild-type and mutant CNGA3 channels}

To determine the impact of the newly identified variant on CNG channel functionality, homomeric as well as heteromeric CNG channels were heterologously expressed in human embryonic kidney 293 (HEK293) cells. Wild-type human CNGA3 (RefSeq NM_001298.2) and CNGB3 (RefSeq NM_019098.3) expression constructs were generated as described previously. ${ }^{29} \mathrm{~A}$ human CNGA3 expression construct harboring the $\mathrm{c} .955 \mathrm{~T}>\mathrm{C}$ variant $\left(\mathrm{CNGA}_{\mathrm{C} 319 \mathrm{R}}\right)$ was obtained via in vitro mutagenesis using the QuikChange Site-Directed Mutagenesis Kit (Agilent Technologies, Santa Clara, CA, USA) and full-length wild-type CNGA3 cDNA cloned into the pcDNA3.1/Zeo $(+)$ vector (Life Technologies, Grand Island, NY, USA) as a template. The correct sequences of the cDNA inserts were confirmed via automated DNA sequencing on an ABI PRISM 3100 Genetic Analyzer (Applied Biosystems).
Column-purified wild-type and p.Cys319Arg mutant expression constructs were employed to transfect HEK293 cells using the Lipofectamine 2000 (Life Technologies) reagent according to the manufacturer's protocol. For calcium imaging, the cells were co-transfected with the cyan fluorescent protein (CFP) expression vector pECFP-C1 (Clontech, Mountain View, CA, USA) to distinguish transfected from untransfected HEK293 cells. For the expression of homomeric channels, we used $8 \mu \mathrm{g}$ of the CNGA3 construct and $2 \mu \mathrm{g}$ of the CFP plasmid, whereas in co-expression experiments, $3 \mu \mathrm{g}$ of the CNGA3 construct and $5 \mu \mathrm{g}$ of the CNGB3 construct were combined with $1.25 \mu \mathrm{g}$ of the CFP plasmid in a 6-well transfection format. For immunocytochemical analyses, HEK293 cells were transfected using $0.5 \mu \mathrm{g}$ of the CNGA3 plasmid and $0.5 \mu \mathrm{g}$ of a plasmid encoding a plasma membrane-bound red fluorescent protein (mHcRED) in a 24-well format. HEK293 cells to be used for western blot experiments were transfected using $15 \mu \mathrm{g}$ of the CNGA3 plasmid in a 6$\mathrm{cm}$ Petri dish. After transfection, the cells were transferred to poly-L-lysinecoated coverslips for calcium imaging and immunocytochemical analyses and incubated at 27 or $37^{\circ} \mathrm{C}$ in DMEM including $10 \%$ fetal bovine serum, $1 \%$ penicillin/streptomycin and 1\% Fungizone (Life Technologies). To stimulate the expression of the transfected plasmids in the calcium imaging and western blot experiments, HEK293 cells were treated with $3 \mu \mathrm{m}$ sodium butyrate (Sigma-Aldrich, Munich, Germany) beginning $24 \mathrm{~h}$ after transfection. Analyses were performed $48 \mathrm{~h}$ after transfection start.

\section{Calcium imaging}

Human wild-type and p.Cys319Arg mutant CNGA3 channels were expressed either alone or together with the wild-type CNGB3 subunit, and the function of the channels was analyzed via calcium imaging using an Olympus BX50WI (Hamburg, Germany) equipped with an imaging system from TILLvisION (TILL Photonics, Graefelfing, Germany). Transfected HEK293 cells were loaded with the $\mathrm{Ca}^{2+}$-sensitive fluorescent dye fura- $2^{30}$ through incubation in an extracellular solution ( $150 \mathrm{~mm} \mathrm{NaCl}, 5 \mathrm{~mm} \mathrm{KCl}, 2 \mathrm{mM} \mathrm{CaCl}_{2}, 2 \mathrm{~mm}$ $\mathrm{MgCl}_{2}, 10 \mathrm{~mm}$ HEPES, and $30 \mathrm{~mm}$ Glucose) containing $4 \mathrm{~mm}$ fura-2acetomethoxyester and $0.04 \%$ Pluronic (Life Technologies) for 20 to $30 \mathrm{~min}$ at 37 or $27^{\circ} \mathrm{C}$. Fluorescence was recorded before and after induction with the membrane-permeable cGMP analog 8-bromoguanosine-3,5'-cyclic monophosphate (8-Br-cGMP; BIOLOG Life Science Institute, Bremen, Germany). The final concentration of 8 -Br-cGMP in the bath solution was $10 \mathrm{~mm}$. At least 20 CFP fluorescence-positive cells were analyzed in at least two separate measurements and from at least two independent transfections.

\section{Western blot and immunocytochemistry analyses}

For western blot experiments, enriched membrane fractions were obtained via centrifugation of HEK293 cell lysates. Membrane fractions of transfected and non-transfected cells were separated through SDS-PAGE (12\% acrylamide gel; $40 \mu \mathrm{g}$ protein per lane) and transferred onto nitrocellulose membranes. The blots were blocked with a $5 \%$ milk powder solution and probed with the antiCNGA3 antiserum SA3899 (1:1000) or anti- $\beta$-actin (1:4000; Chemicon/ Millipore, Billerica, MA, USA) as a loading control, followed by an anti-rabbit IgG horseradish peroxidase-linked secondary antibody (1:4000; Amersham Bio-sciences, Arlington Heights, IL, USA) or anti-mouse IgG horseradish peroxidase-linked secondary antibody (1:10000; Calbiochem/Merck, Darmstadt, Germany), respectively. SA3899 is a rabbit polyclonal antiserum raised against the C-terminus (p.Ile605 to Gln694) of the human CNGA3 protein that was established in our group.

Immunocytochemical analyses of transfected HEK293 cells were performed to analyze the integration of mutant channels into the plasma membrane. HEK293 cells were co-transfected with a plasmid encoding a red-fluorescent protein ( $\mathrm{mHcRed}$ ) that is targeted to the plasma membrane via an N-termina palmitoylation signal to label the plasma membrane. The coding sequence of GFP in pCAG-mGFP ${ }^{31}$ was replaced with the coding sequence of HcRed from pHcRed1-1N1 (Clontech). Immunocytochemical staining was performed $48 \mathrm{~h}$ after transfection as described previously. ${ }^{32} \mathrm{CNG}$ channels were detected with the anti-CNGA3 primary antibody SA3899 (1:250) and an Alexa-488 coupled goat anti-rabbit antibody (1:250; Life Technologies). Image acquisition was performed using the Axio Imager Z1 System with Apotome (Carl Zeiss AG, Oberkochen, Germany). Z-stacks of labeled cells were recorded with a distance 
of $0.15 \mu \mathrm{m}$ at $\times 63$ magnification. The image data were analyzed using Axio Vision Rel. 4.8 (Carl Zeiss AG) and ImageJ 1.43m (Rasband WS, ImageJ, National Institutes of Health, Bethesda, MD, USA).

Tools from Image $1.43 \mathrm{~m}$ (Rasband WS, ImageJ) were used to assess the degree of colocalization of the wild-type and mutant channels with the plasma membrane. The background fluorescence from CNGA3 staining and mHcRed fluorescence was subtracted using the 'BG subtraction from ROI' plug-in. Subsequently, the cell membrane of a cell that expressed CNG channels was selected using the region of interest tool, and the Manders' overlap coefficient was calculated with the 'Manders coefficients' plug-in. For the wild-type and p.Cys319Arg mutant channels, at least 10 cells were analyzed. The data are presented as the mean $\pm S D$. For statistical evaluation, the Mann-Whitney rank sum test was performed using MYSTAT 12 Version 12.02.00 (SYSTAT Inc., Chicago, IL, USA).

\section{RESULTS}

\section{Clinical evaluation revealed CRD in family PKAB157}

We enrolled a large, consanguineous Pakistani family (PKAB157) with six affected siblings, whose ages range from 4 to 17 years (Figure 1). Detailed clinical evaluation of the affected individuals of family PKAB157 revealed juvenile CRD with maculopathy (Table 1). Funduscopic imaging of both eyes revealed macular atrophy of varying degrees in all of the affected patients (Figure 2). The observed macular atrophy was centered on the fovea and included the retina, retinal pigment epithelium (RPE) and choroid, with preservation of larger and deep choroidal vessels (Figure 2). Spectral domain optical coherence tomography (SD-OCT) horizontal raster and foveal scans revealed retinal and RPE thinning with transmission defects in normal-thickness choroids or irregular RPE thickening at the fovea, surrounded on each side by disruption of the inner segment ellipsoid band and outer retinal thinning (Supplementary Figure 1).

Despite the clinical appearance of localized, central retinal degeneration, full-field ERG demonstrated a pattern of cone (photopic) responses that were markedly reduced or absent, suggesting widespread retinal dysfunction (Figure 3). Rod (scotopic) responses were observed in the younger affected individuals (eg, IV:4; Figure 3) but were significantly diminished in the older affected siblings, consistent with a diagnosis of CRD. Color vision testing using the panel D-15 test revealed a diffuse color vision deficit among the affected individuals (Supplementary Figure 2).

\section{A missense variant in CNGA3 causes CRD in family PKAB157}

Exome sequencing in siblings IV:1 and IV:4 revealed a c.955T >C transition alteration in exon 7 of CNGA3 (GenBank NM_001298.2) that co-segregated with the CRD phenotype in family PKAB157 (Figure 1; Table 2). The c.955T $>\mathrm{C}$ change is predicted to cause the substitution of cysteine for arginine at position 319 (p.(Cys319Arg)) of the polypeptide. This residue is located in the fifth transmembrane (S5) region of the CNGA3 channel subunit (Figure 4a). No carriers of the c.955T $>\mathrm{C}$ variant were found among 552 ancestry-matched control chromosomes, in the 1000 Genomes database or in the 6500 individuals who are listed in the NHLBI-ESP variant database. ${ }^{21,22}$ Segregation analysis confirmed biparental transmission of the variant (Figure 1). A statistically significant two-point LOD score of 3.14 at recombination fraction $\theta=0$ was observed for $C N G A 3$-linked STR marker D2S2222, further supporting the linkage between the CRD phenotype and CNGA3 on chromosome 2q11.2 in family PKAB157.

\section{Bioinformatics analyses predict CNGA3 misfolding due to the p.(Cys319Arg) variant}

Amino-acid alignments of several CNGA3 orthologs and human CNG alpha and beta subunits indicate high conservation of cysteine 319 (Figures $4 \mathrm{~b}$ and c). Polyphen-2 (probability score 1.000), SNPs3D ( -3.61$)$ and MutationTaster (0.999) prediction programs suggested that the p.(Cys319Arg) alteration was deleterious. The HOPE prediction program suggested that due to its positive charge, size, and lower hydrophobicity compared with the wild-type residue, the substitution of an arginine at position 319 is predicted to disturb ionic and hydrophobic interactions with the other transmembrane helices or

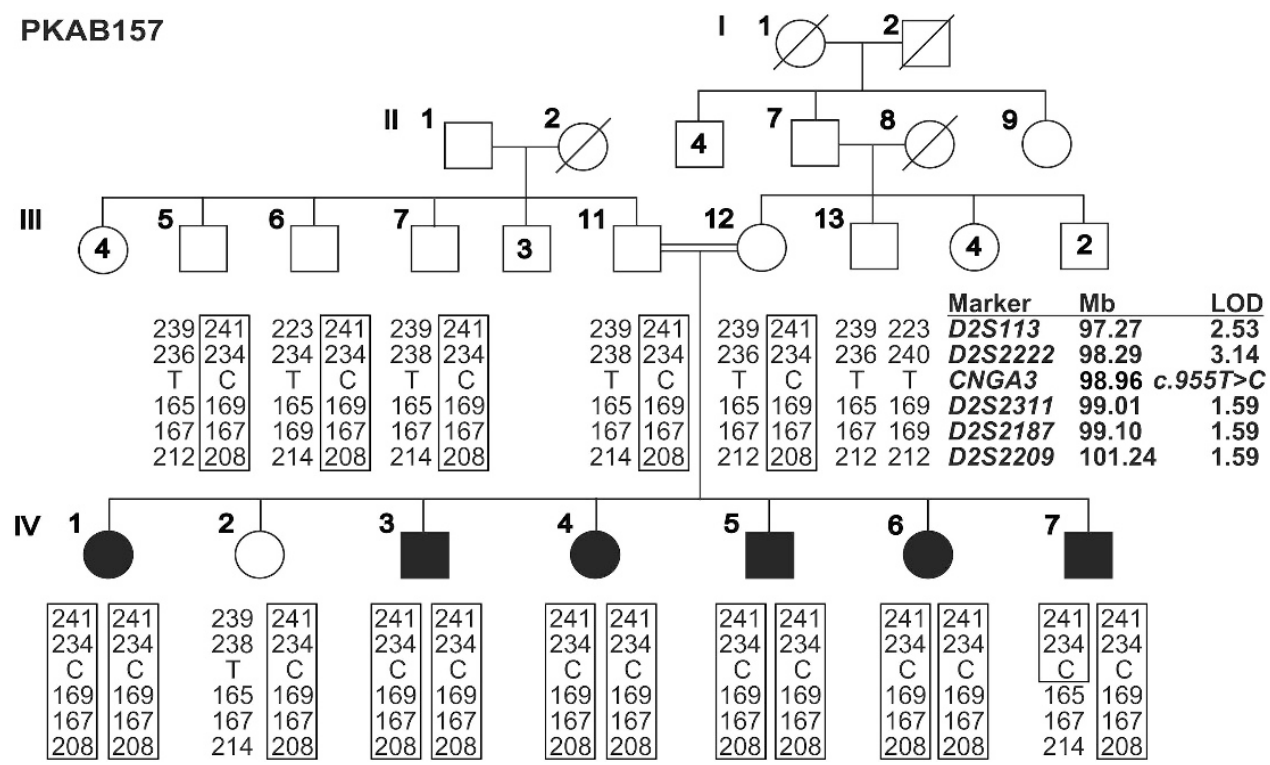

Figure 1 Segregation of CNGA3 alleles in family PKAB157. Filled and empty symbols represent affected and unaffected individuals, respectively. Double lines indicate consanguineous marriages. Chromosome 2q11.2-linked STR markers were used for haplotype analysis. The disease haplotype is boxed. The STR markers and physical map positions in megabases (Mb, February 2009 human reference sequence GRCh37, hg19) are shown to the right of the pedigree. The maximum two-point LOD scores for chromosome 2q11.2-linked STR markers are also given. The genotypes (wild-type, heterozygous and homozygous mutant) of the CNGA3 mutant allele are also shown for each of the participating family members. 
Table 1 Summary of clinical features of cone-rod dystrophy segregating in family PKAB157

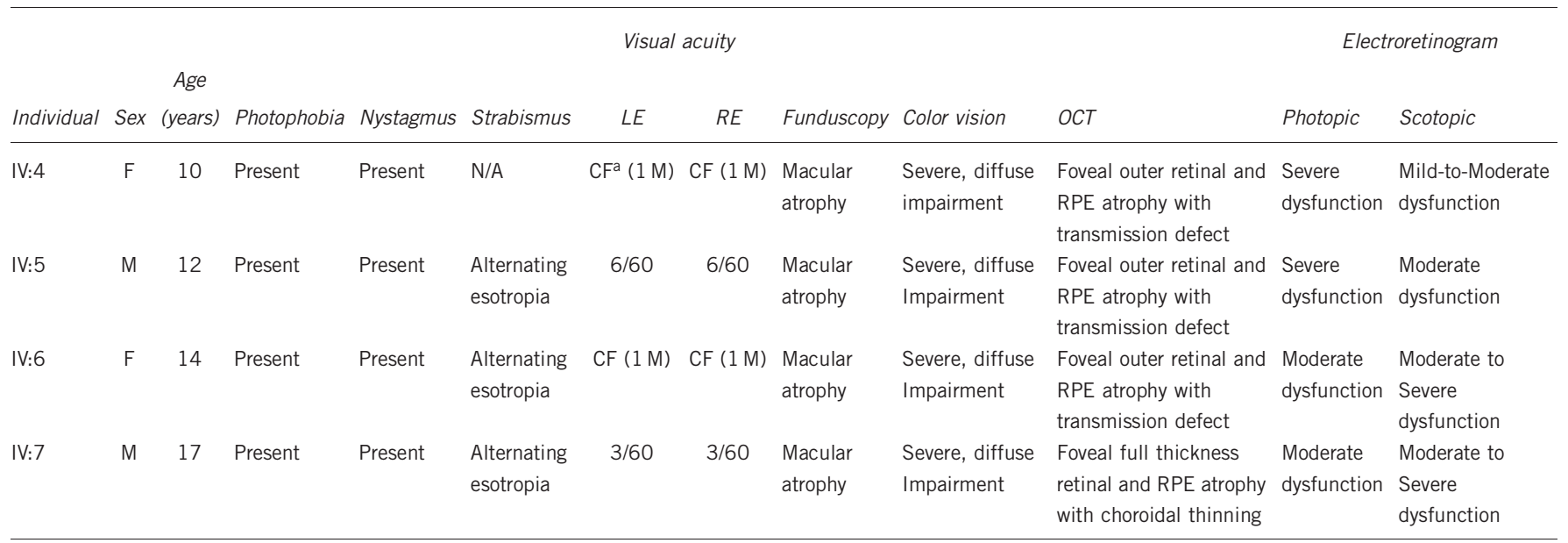

${ }^{\mathrm{a} C F}(1 \mathrm{M})$, Counting fingers (1 meter); N/A, not available.
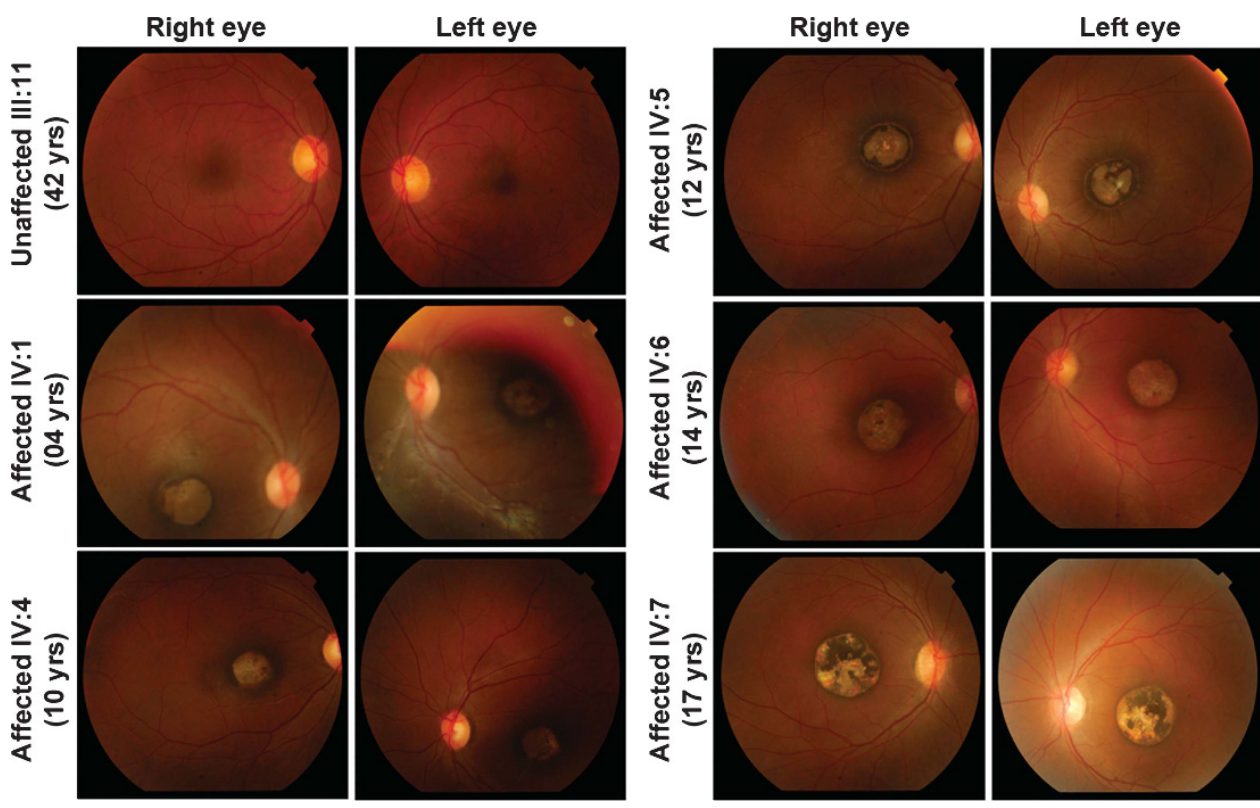

Figure 2 Funduscopic imaging revealed macular degeneration in affected individuals from family PKAB157. Color photographs of the fundus from both eyes demonstrate macular atrophy of varying degrees in all affected individuals. This macular atrophy was centered on the fovea and included the retina, RPE and choroid, with preservation of larger, deep choroidal vessels. The areas of atrophy were surrounded by a ring of RPE hyperplasia and, in individual $\mathrm{IV}: 5$, radially oriented retinal striae.

with membrane lipids and could therefore disrupt the protein topology, potentially, resulting in protein misfolding.

The channel activity of CNGA3 is significantly diminished due to the p.Cys319Arg variant

To determine the impact of the p.Cys319Arg variant on the channel function of CNGA3, we performed a ligand-induced calcium permeability assay using human embryonic kidney (HEK293) cells heterologously expressing either homo-oligomeric (only CNGA3) or hetero-oligomeric (CNGA3 + CNGB3) channels. In contrast to the wild-type protein, the CNGA3 channel harboring the p.Cys319Arg alteration did not mediate any 8-Br-cGMP-induced calcium influx when expressed either alone or together with the wild-type CNGB3 subunit (Table 3). Reducing the culture temperature of transfected
HEK293 cells, which is a method that has previously been shown to enhance the folding and/or trafficking of mutant CNG channels, ${ }^{32,33}$ did not improve the functionality of mutant homomeric or heteromeric channels (Table 3 ).

The p.Cys319Arg variant affects the stability of the CNGA3 protein To confirm the expression of the CNGA3 mutant channel $\left(\mathrm{CNGA} 3_{\mathrm{C} 319 \mathrm{R}}\right)$ in transiently transfected HEK293 cells, we performed western blot analysis using enriched membrane fractions and an anti-CNGA3 antibody. The levels of the $\mathrm{CNGA}_{\mathrm{C} 319 \mathrm{R}}$ protein were comparatively lower than the wild-type protein, possibly as a result of reduced protein stability (Figure $5 \mathrm{a}$ ). To exclude false negative results due to a hidden variant in the expression construct, two clones from two independent in vitro mutagenesis experiments were analyzed. 
Scotopic Rod Response

White flash (-25bB)
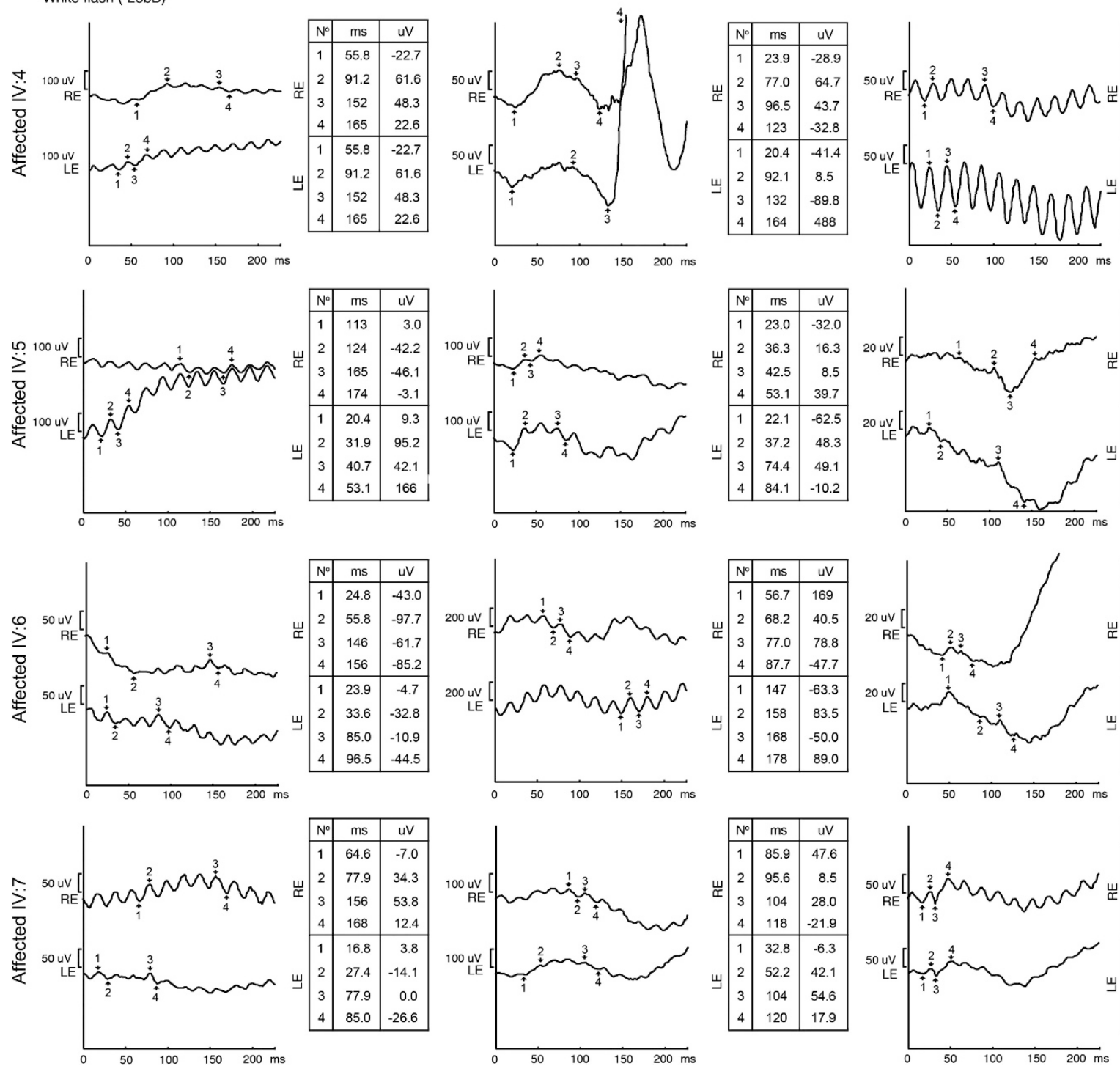

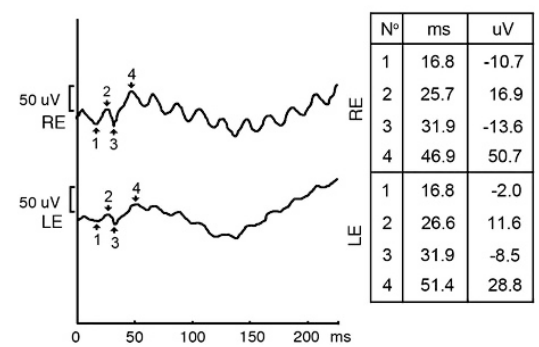

Figure 3 Full-field ERG revealed defective rod and cone responses in affected individuals of family PKAB157. Full-field ERG demonstrated a progressive reduction in amplitude and increased implicit times associated with age in all phases of testing. Despite a wandering baseline and $60 \mathrm{~Hz}$ noise, a pattern of greater cone than rod dysfunction emerged among the younger affected individuals that was nearly extinguished in older affected siblings.

Next, we performed immunocytochemistry experiments to determine whether the p.Cys319Arg variant affected the integration of the mutant channel into the plasma membrane (Figures $5 \mathrm{~b}$ and c). For this purpose, HEK293 cells were co-transfected with a plasmid encoding a red-fluorescent protein ( $\mathrm{mHcRed}$ ) targeted to the plasma membrane. To quantify the extent of colocalization of the $\mathrm{CNGA}{ }_{\mathrm{C} 319 \mathrm{R}}$ mutant channel with the plasma membrane, we calculated the Manders' overlap coefficient. ${ }^{34}$ In comparison with the wild-type protein, the homomeric $\mathrm{CNGA}_{\mathrm{C} 319 \mathrm{R}}$ mutant channel showed a significantly reduced Manders' overlap coefficient, indicating that the integration of $\mathrm{CNGA}_{\mathrm{C} 319 \mathrm{R}}$ channels into the plasma membrane was reduced due to impaired protein folding and/ or trafficking (Figure 5c). Taken together with calcium imaging results, our functional analyses revealed that in vitro the p.Cys319Arg alteration in the S5 region severely affects the stability and function of the encoded mutant CNGA3 channel.

\section{DISCUSSION}

Previous studies have reported three cases of CD due to pathogenic variants of CNGA3. ${ }^{14}$ The clinical features of these individuals included abnormal color vision, photophobia, nystagmus and reduced or absent cone ERG responses. ${ }^{14}$ In this study, we identified a pathogenic missense variant in CNGA3 that cosegregates with a CRD phenotype in family PKAB157. The affected individuals from family PKAB157 presented similar cone photoreceptor abnormalities as reported for individuals with CD. ${ }^{14}$ In addition, we performed funduscopic examinations and measured scotopic ERG responses, which further suggested rod dysfunction in affected individuals of family PKAB157. It should be noted that detailed description of rod photoreceptors function was not reported for three individuals with CNGA3-associated cone dystrophies, ${ }^{14}$ thereby preventing us from further comparison. Nevertheless, due to the very early onset rod dysfunction and macular atrophy observed 
in some patients (eg, individual IV:1), the phenotype segregating in family PKAB157 was classified as CRD. ${ }^{1,13}$ The CRD phenotype segregating in family PKAB157 shows progression in the severity of the disease with age. Imaging evaluations of all of the affected individuals homozygous for the c.955T $>\mathrm{C}$ variant revealed macular atrophy. Such findings in the fundus have frequently been described as 'macular coloboma', although this term is inappropriate, as their appearance bears no relation to defects of optic cup closure during embryonic development. In the obtained ERG responses, the rod amplitudes and implicit times (in addition to the cones) were also progressively more abnormal, especially in the older affected siblings, which suggest age-dependent progression to CRD phenotype.

The phenotype observed in the affected individuals from family PKAB157 is consistent with the consequences to retinal function observed in Cnga3 knockout mice, in which loss of cone function also leads to secondary rod dysfunction and degeneration. ${ }^{35}$ Due to the

Table 2 Summary of exome sequencing analysis of PKAB157affected individuals

\begin{tabular}{lcc}
\hline & IV:1 & IV:4 \\
\hline Total mapped reads (Gbp) & 5.33 & 6.14 \\
Target coverage (\%) & 96.75 & 97.42 \\
Mean depth of coverage $(\times)$ & 65 & 77 \\
Depth $\geq 20 \times$ of coverage (\%) & 86.87 & 89.30 \\
Total changes & 54963 & 55971 \\
Changes not in db135 (MAF $\geq 0.01$ ) & 5039 & 5150 \\
Non-synonymous/Splice site/insertions/deletions & 701 & 749 \\
Changes not found in Pakistani control samples & 534 & 577 \\
Genes with homozygous/compound heterozygous changes & 23 & 26 \\
Changes not found in 1000 genome or NHLBI ESP & 15 & 24 \\
Changes predicted to be pathogenic ${ }^{\text {a }}$ & 2 & 3 \\
Genes with nucleotide variations in both samples & 1 & 1 \\
Changes co-segregating with the phenotype in family & 1 & 1 \\
\hline
\end{tabular}

aPathogenicity predicted by at least two of the three prediction programs: Polyphen-2, SNPs3D and MutationTaster. observed cone and rod abnormalities, Cnga3 null mice are classified as a model for CRD. ${ }^{35}$ Several possible explanations have been proposed for the secondary rod dysfunction found in Cnga3 null mice, including attenuated transmission from rod to rod bipolar cells, remodeling of the neuronal circuitry, impaired ribbon synapse integrity or aberrant gap junctional coupling of cones and rods. ${ }^{35}$ It is intriguing that in humans, different alleles of CNGA3 cause clinically distinct phenotypes, achromatopsia, CD, CRD or LCA. One possibility is that the genetic background modifies the phenotypic outcome of CNGA3 variants. Alternatively, differences in mutant alleles of $C N G A 3$ may directly account for the different phenotypic outcomes. Knock-in alleles of mouse Cnga3 engineered to model human variants could aid in addressing these questions.

The native cone cyclic nucleotide-gated (CNG) channel is composed of three CNGA3 subunits and one CNGB3 subunit, ${ }^{36}$ and displays a crucial function in cone phototransduction. ${ }^{37-39}$ The CNGA3 and CNGB3 subunits are structurally similar, being composed of six transmembrane domains (S1-S6), a pore-forming region, a cyclic nucleotide-binding domain and a C-linker region (Figure 4a). While CNGA3 alone is sufficient to form functional CNG channels in heterologous expression systems, CNGB3 requires co-expression of the CNGA3 subunit. ${ }^{40,41}$ Although 81 different variants of CNGA3 have been documented to date, only 2 known

Table 3 Calcium imaging experiments

\begin{tabular}{lccccc}
\hline & \multicolumn{2}{c}{$37^{\circ} \mathrm{C}$} & & \multicolumn{2}{c}{$27^{\circ} \mathrm{C}$} \\
\cline { 2 - 3 } \cline { 5 - 6 } & Fraction (\%) & $n^{\mathrm{a}}$ & & Fraction (\%) & $n^{\mathrm{a}}$ \\
\hline CNGA3 $_{\text {WT }}$ & $73.8 \pm 10.0$ & 85 & & $85.4 \pm 13.9$ & 57 \\
CNGA3 $_{\text {C319R }}$ & $0 \pm 0$ & 58 & & $0 \pm 0$ & 39 \\
CNGA3 $_{W T}+$ CNGB3 & $67.1 \pm 15.9$ & 81 & & $76.2 \pm 16.0$ & 29 \\
CNGA3 $_{\text {C319R }}+$ CNGB3 & $0 \pm 0$ & 55 & & $0 \pm 0$ & 20 \\
\hline
\end{tabular}

Summarized is the fraction of CFP-positive cells that showed an influx of calcium upon stimulation with the ligand 8-Br-cGMP. anumber of analyzed CFP-positive cells.

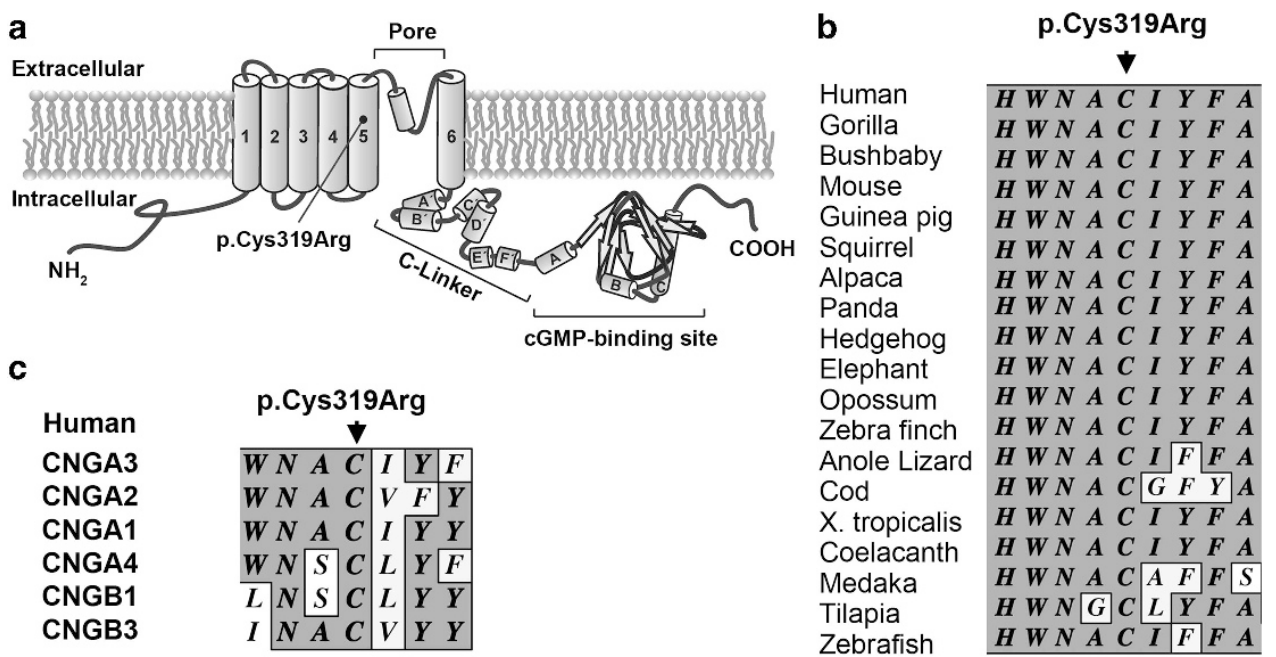

Figure 4 CNGA3 protein. (a) Topological model of the CNGA3 protein, which consists of six transmembrane helices (S1-S6), a pore region and a C-linker connecting S6 and the cyclic nucleotide-binding domain. The filled circle indicates the location of the p.(Cys319Arg) variant. (b) ClustalW multiple aminoacid sequence alignment of CNGA3 orthologs shows that p.Cys319 is highly conserved across species (shaded background, same amino acid; light background, non-conserved amino acids). (c) ClustalW multiple amino-acid sequence alignment of human CNGA3 homologs shows conservation of p.Cys319 residues among human cyclic nucleotide-gated channel subunits. 
a

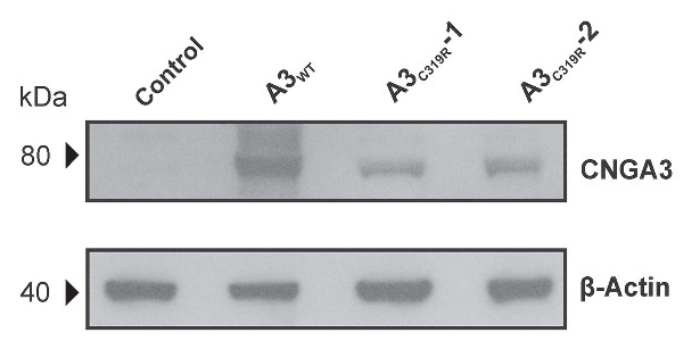

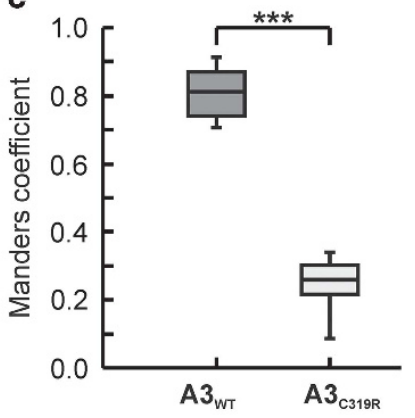

b
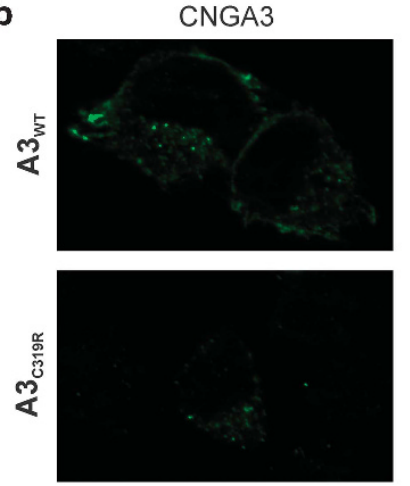

mHcRed
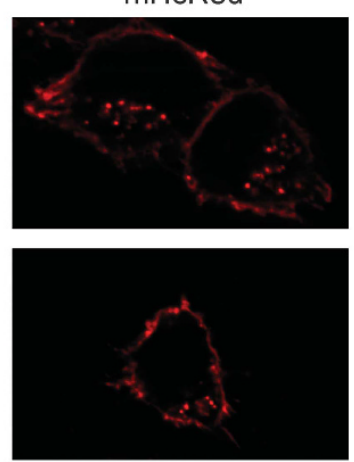

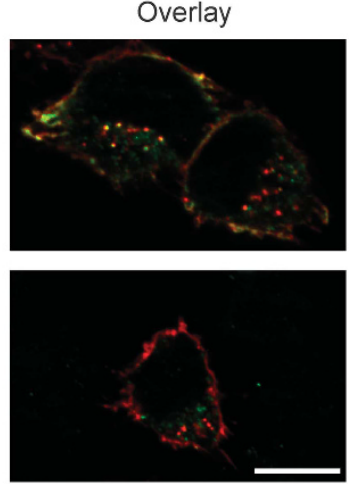

Figure 5 Expression and localization of the p.Cys319Arg mutant CNGA3 protein in HEK293 cells. (a) Western blot analysis revealed reduced expression of the $\mathrm{CNGA}_{\mathrm{C} 319 \mathrm{R}}$ mutant protein. Western blots of cell membrane fractions from HEK293 cells expressing wild-type (CNGA3 ${ }_{W T}$ ) or mutant (CNGA3 ${ } 319 R^{-1}{ }$,

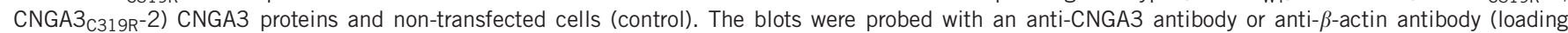
control). A reduced level of the $\mathrm{CNGA3}_{\mathrm{C} 319 \mathrm{R}}$ mutant protein was observed using both independently generated constructs. (b and $\left.\mathbf{c}\right)$ Subcellular localization of mutant and wild-type CNGA3 channels in transfected HEK293 cells. Scale bar represents $10 \mu \mathrm{m}$. (b) CNGA3 subunits were immunolabeled in green with an anti-CNGA3 primary antibody and an Alexa-488-coupled secondary antibody. As a plasma membrane marker, HEK293 cells were co-transfected with a plasmid encoding a red-fluorescent protein ( $\mathrm{mHcRed}$ ) that is targeted to the plasma membrane via an $\mathrm{N}$-terminal palmitoylation signal. Compared with the wild-type protein, significantly reduced colocalization of the CNGA3 ${ }_{C 319 R}$ mutant channel and plasma membrane was observed. (c) The Manders' coefficient was used to quantify the colocalization of the red and green staining at the plasma membrane, further confirming the statistically significantly $(* * * P<0.001)$ reduced expression of the CNGA3 channel harboring p.Cys319Arg variant at the plasma membrane.

variants, c.934_936delATC (p.(Ile312del)) and c.947G >A (p. $\left.\left(\operatorname{Trp} 316^{\star}\right)\right)$ have been found in the fifth transmembrane domain (Figure $4 \mathrm{a}$ ) of the CNGA3 protein. ${ }^{38}$ The p.(Cys319Arg) allele segregating in family PKAB157 is the first homozygous missense variant located in the S5 region (Figure 4a). The results of our in vitro functional characterizations support the pathogenic nature of p.Cys319Arg change, which might not mediate any cone function under physiological conditions. In young affected individuals from family PKAB157, residual cone function was intact, despite the fact that our in vitro functional studies suggested that p.Cys319Arg is a loss-of-function allele of CNGA3. This apparent lack of correlation with the phenotype observed in the patients could be due to an insufficient sensitivity of our in vitro HEK293 cell-based functional system or basic differences compared with native cone photoreceptor cells. We therefore reason that a few mutant channels might have escaped the quality control process in early cones and be sufficient to carry out residual cone responses in the young affected individuals, after which these cones rapidly degenerate with age.

Numerous CNGA3 variants, causing achromatopsia, have been assessed functionally upon heterologous expression in Xenopus oocytes or HEK293 cells with the majority of mutant channels being non-functional in calcium imaging or patch clamp experiments, ${ }^{29,32,33,42-45}$ - similar to what has been observed for p.Cys319Arg mutant channels. Besides affecting the apparent ligand sensitivity, impaired protein folding and/or trafficking resulting in a decreased channel density at the cell membrane is a common consequence of variants in the CNGA3 subunit. $32,33,42,43,45$ Immunocytochemical analyses performed in this study demonstrated that the p.Cys319Arg variant also leads to a disturbed surface expression. For some of the CNGA3 variants, it was shown that this folding/trafficking defect could be rescued by the presence of the wild-type CNGB3 subunit in heteromeric channels or by lowering the cultivation temperature of the corresponding heterologous expression system..$^{29,32,33,42-45}$ Both approaches were also applied in the case of the p.Cys319Arg mutant channels, but here no improvement of channel functionality could be observed in calcium imaging experiments. As shown in this study, the alteration in p.Cys319Arg also affects protein stability, an observation which has so far only been described for two pore variants and one variant located in transmembrane domain 4 of $\mathrm{CNGA} 3,{ }^{42,43}$ representing another mechanism how CNGA3 variants impair channel functionality.

In summary, our data demonstrate that the c.955T $>C$ variant in CNGA3 causes early onset progressive CRD with maculopathy. Functional analysis of this transmembrane helix S5 mutant reveals instability of the protein and impaired surface expression, indicating the importance of S5 for the structural and functional integrity of the cone CNG channel. Our clinical and molecular analysis of CNGA3 alleles may prove useful for future genetic diagnosis and counseling as well as for molecular epidemiology studies of cone-rod dystrophies. 


\section{CONFLICT OF INTEREST}

The authors declare no conflict of interest.

\section{ACKNOWLEDGEMENTS}

We thank the families for their participation and cooperation. We also thank Thomas Jaworek and Muhammad Asim Bhatti for technical assistance. The work in Pakistan was supported by a research grant (HEC-1262) to RSS from the Higher Education Commission of Islamabad. This work was supported through a grant from the German Ministry for Education and Research (BMBF; IonNeuroNet, FZ. 01GM1105A to BW) and the National Institutes of Health - National Institute on Deafness and Other Communication Disorders grant (R01DC012564 to ZMA). ZMA is also a recipient of an RPB Career Development Award.

1 Hamel CP: Cone rod dystrophies. Orphanet J Rare Dis 2007; 2: 7.

2 Michaelides M, Hardcastle AJ, Hunt DM, Moore AT: Progressive cone and cone-rod dystrophies: phenotypes and underlying molecular genetic basis. Surv Ophthalmol 2006; 51: 232-258.

3 Michaelides M, Hunt DM, Moore AT: The cone dysfunction syndromes. Br J Ophthalmo 2004; 88: 291-297.

4 Cremers FP, van de Pol DJ, van Driel M et al: Autosomal recessive retinitis pigmentosa and cone-rod dystrophy caused by splice site mutations in the Stargardt's disease gene ABCR. Hum Mol Genet 1998; 7: 355-362.

5 Parry DA, Toomes C, Bida L et al: Loss of the metalloprotease ADAM9 leads to cone-rod dystrophy in humans and retinal degeneration in mice. Am J Hum Genet 2009; 84: 683-691.

6 Estrada-Cuzcano A, Neveling K, Kohl S et al: Mutations in C8orf37, encoding a ciliary protein, are associated with autosomal-recessive retinal dystrophies with early macular involvement. Am J Hum Genet 2012; 90: 102-109.

7 Tuson M, Marfany G, Gonzalez-Duarte R: Mutation of CERKL, a novel human ceramide kinase gene, causes autosomal recessive retinitis pigmentosa (RP26). Am J Hum Genet 2004; 74: 128-138.

8 Abd El-Aziz MM, Barragan I, O'Driscoll CA et al: EYS, encoding an ortholog of Drosophila spacemaker, is mutated in autosomal recessive retinitis pigmentosa. Nat Genet 2008; 40: 1285-1287.

9 Hameed A, Abid A, Aziz A, Ismail M, Mehdi SQ, Khaliq S: Evidence of RPGRIP1 gene mutations associated with recessive cone-rod dystrophy. J Med Genet 2003; 40 616-619.

10 Roosing S, Rohrschneider K, Beryozkin A et al: Mutations in RAB28, encoding a farnesylated small GTPase, are associated with autosomal-recessive cone-rod dystrophy. Am J Hum Genet 2013; 93: 110-117.

11 Roosing S, van den Born LI, Hoyng CB et al: Maternal uniparental isodisomy of chromosome 6 reveals a TULP1 mutation as a novel cause of cone dysfunction. Ophthalmology 2013; 120: 1239-1246.

12 Littink KW, Koenekoop RK, van den Born LI et al: Homozygosity mapping in patients with cone-rod dystrophy: novel mutations and clinical characterizations. Invest Ophthalmol Vis Sci 2010; 51: 5943-5951.

13 Thiadens AA, Phan TM, Zekveld-Vroon RC et al: Clinical course, genetic etiology, and visual outcome in cone and cone-rod dystrophy. Ophthalmology 2012; 119: 819-826.

14 Thiadens AA, Roosing S, Collin RW et al: Comprehensive analysis of the achromatopsia genes CNGA3 and CNGB3 in progressive cone dystrophy. Ophthalmology 2010; 117 825-830, e821.

15 Wang $\mathrm{X}$, Wang $\mathrm{H}$, Cao $\mathrm{M}$ et al: Whole-exome sequencing identifies ALMS1, IQCB1, CNGA3, and MYO7A mutations in patients with Leber congenital amaurosis. Hum Mutat 2011; 32: 1450-1459.

16 Vincent A, Wright T, Billingsley G, Westall C, Heon E: Oligocone trichromacy is part of the spectrum of CNGA3-related cone system disorders. Ophthalmic Genet 2011; 32: 107-113.

17 DePristo MA, Banks E, Poplin R et al: A framework for variation discovery and genotyping using next-generation DNA sequencing data. Nat Genet 2011; 43 491-498.

$18 \mathrm{Li} \mathrm{H}$, Durbin R: Fast and accurate short read alignment with Burrows-Wheeler transform. Bioinformatics 2009; 25: 1754-1760.
19 Harismendy O, Frazer K: Method for improving sequence coverage uniformity of targeted genomic intervals amplified by LR-PCR using Illumina GA sequencing-bysynthesis technology. BioTechniques 2009; 46: 229-231.

20 Harismendy O, Frazer KA: Elucidating the role of 8 q24 in colorectal cancer. Nat Genet 2009; 41: 868-869.

21 Abecasis GR, Auton A, Brooks LD et al: An integrated map of genetic variation from 1092 human genomes. Nature 2012; 491: 56-65.

22 Fu W, O'Connor TD, Jun G et al: Analysis of 6515 exomes reveals the recent origin of most human protein-coding variants. Nature 2013; 493: 216-220.

23 Ahmed ZM, Riazuddin S, Bernstein SL et al: Mutations of the protocadherin gene PCDH15 cause Usher syndrome type 1F. Am J Hum Genet 2001; 69: 25-34.

24 Schaffer AA: Faster linkage analysis computations for pedigrees with loops or unused alleles. Hum Hered 1996; 46: 226-235.

25 Adzhubei IA, Schmidt S, Peshkin L et al: A method and server for predicting damaging missense mutations. Nat Methods 2010; 7: 248-249.

26 Yue P, Melamud E, Moult J: SNPs3D: candidate gene and SNP selection for association studies. BMC Bioinformatics 2006; 7: 166

27 Schwarz JM, Rodelsperger C, Schuelke M, Seelow D: MutationTaster evaluates disease-causing potential of sequence alterations. Nat Methods 2010; 7 575-576.

28 Venselaar H, Te Beek TA, Kuipers RK, Hekkelman ML, Vriend G: Protein structure analysis of mutations causing inheritable diseases. An e-Science approach with life scientist friendly interfaces. BMC Bioinformatics 2010; 11: 548.

29 Trankner D, Jagle H, Kohl S et al: Molecular basis of an inherited form of incomplete achromatopsia. J Neurosci 2004; 24: 138-147.

30 Grynkiewicz G, Poenie M, Tsien RY: A new generation of $\mathrm{Ca} 2+$ indicators with greatly improved fluorescence properties. J Biol Chem 1985; 260: 3440-3450.

31 Matsuda T, Cepko CL: Controlled expression of transgenes introduced by in vivo electroporation. Proc Natl Acad Sci USA 2007; 104: 1027-1032.

32 Reuter P, Koeppen K, Ladewig T, Kohl S, Baumann B, Wissinger B: Mutations in CNGA3 impair trafficking or function of cone cyclic nucleotide-gated channels, resulting in achromatopsia. Hum Mutat 2008; 29: 1228-1236.

33 Koeppen K, Reuter P, Kohl S, Baumann B, Ladewig T, Wissinger B: Functional analysis of human CNGA3 mutations associated with colour blindness suggests impaired surface expression of channel mutants A3(R427C) and A3(R563C). Eur J Neurosci 2008; 27: 2391-2401.

34 Zinchuk V, Grossenbacher-Zinchuk O: Recent advances in quantitative colocalization analysis: focus on neuroscience. Prog Histochem Cytochem 2009; 44: 125-172.

$35 \mathrm{Xu} \mathrm{J}$, Morris LM, Michalakis S et al: CNGA3 deficiency affects cone synaptic termina structure and function and leads to secondary rod dysfunction and degeneration. Invest Ophthalmol Vis Sci 2012; 53: 1117-1129.

36 Shuart NG, Haitin Y, Camp SS, Black KD, Zagotta WN: Molecular mechanism for 3:1 subunit stoichiometry of rod cyclic nucleotide-gated ion channels. Nat Commun 2011; 2. 457.

37 Kohl S, Baumann B, Broghammer M et al: Mutations in the CNGB3 gene encoding the beta-subunit of the cone photoreceptor cGMP-gated channel are responsible for achromatopsia (ACHM3) linked to chromosome 8q21. Hum Mol Genet 2000; 9: 2107-2116

38 Wissinger B, Gamer D, Jagle $\mathrm{H}$ et al: CNGA3 mutations in hereditary cone photoreceptor disorders. Am J Hum Genet 2001; 69: 722-737.

39 Peng C, Rich ED, Varnum MD: Subunit configuration of heteromeric cone cyclic nucleotide-gated channels. Neuron 2004; 42: 401-410.

40 Bonigk W, Altenhofen W, Muller F et al: Rod and cone photoreceptor cells express distinct genes for cGMP-gated channels. Neuron 1993; 10: 865-877.

41 Gerstner A, Zong X, Hofmann F, Biel M: Molecular cloning and functional characterization of a new modulatory cyclic nucleotide-gated channel subunit from mouse retina. J Neurosci 2000; 20: 1324-1332.

42 Koeppen K, Reuter P, Ladewig T et al: Dissecting the pathogenic mechanisms of mutations in the pore region of the human cone photoreceptor cyclic nucleotide-gated channel. Hum Mutat 2010; 31: 830-839.

43 Liu C, Varnum MD: Functional consequences of progressive cone dystrophy-associated mutations in the human cone photoreceptor cyclic nucleotide-gated channel CNGA3 subunit. Am J Physiol Cell Physiol 2005; 289: C187-C198.

44 Muraki-Oda S, Toyoda F, Okada A et al: Functional analysis of rod monochromacyassociated missense mutations in the CNGA3 subunit of the cone photoreceptor cGMP-gated channel. Biochem Biophys Res Commun 2007; 362: 88-93.

45 Patel KA, Bartoli KM, Fandino RA et al: Transmembrane S1 mutations in CNGA3 from achromatopsia 2 patients cause loss of function and impaired cellular trafficking of the cone CNG channel. Invest Ophthalmol Vis Sci 2005; 46: 2282-2290.

Supplementary Information accompanies this paper on European Journal of Human Genetics website (http://www.nature.com/ejhg) 